\title{
The association between coronary slow flow and platelet distribution width among patients with stable angina pectoris
}

\author{
Ferhat Ozyurtlu ${ }^{1}$, Veysel Yavuz ${ }^{2}$, Nurullah Cetin ${ }^{3}$, Halit Acet $^{4}$, Erkan Ayhan ${ }^{5}$, Turgay Isik \\ ${ }^{1}$ Special Grand Medical Hospital, Manisa, Turkey \\ ${ }^{2}$ Akhisar State Hospital, Manisa, Turkey \\ ${ }^{3}$ Ercis State Hospital, Van, Turkey \\ ${ }^{4}$ Department of Cardiology, Dicle University Faculty of Medicine, Diyarbakır, Turkey \\ ${ }^{5}$ Department of Cardiology, Balıkesir University Faculty of Medicine, Balıkesir, Turkey
}

Postep Kardiol Inter 2014; 10, 3 (37): 161-165

DOI: $10.5114 /$ pwki.2014.45142

\begin{abstract}
A bstract
Introduction: Coronary slow flow (CSF) is an angiographic phenomenon characterised by the delay of distal vessel opacification in the absence of significant stenosis of the epicardial coronary arteries. Some of the factors playing a role in CSF pathophysiology are increased thrombogenic activity and inflammation.

Aim: To examine the relationship between platelet distribution width (PDW) and CSF.

Material and methods: Taking into consideration the exclusion criteria, 136 patients with CSF and 152 patients with normal coronary angiographies (control group) were included in the study. The association between thrombolysis infarction frame count (TFC) in myocardial and laboratory and other clinical parameters were evaluated.

Results: The stated parameters were significantly higher in the group with CSF than in the normal coronary angiography group (control group). The PDW (16.6 \pm 0.7 vs. $16.4 \pm 0.6, p=0.002)$, neutrophil lymphocyte ratio (NLR) $(3.1 \pm 3.4$ vs. $2.4 \pm 1.1, p=0.027)$, haemoglobin $(\mathrm{Hb})(14.1 \pm 1.3$ vs. $14.7 \pm 1.1, p<0.001)$, and red cell distribution width (RDW) $(13.6 \pm 0.7$ vs. $14.1 \pm 2.8, p=0.026)$ were significantly higher in the CSF group than in the control group. Moreover, our study showed that PDW > 16.15 and Hb $>13.75$ were predictors of the presence of CSF with sensitivities of $83 \%$ and $73 \%$ and specificities of $40 \%$ and $42 \%$, respectively.

Conclusions: This study has demonstrated that compared to normal coronary flow, PDW, Hb, NLR, and RDW are significantly higher in CSF patients. We believe that further studies are needed to clarify the role of PDW and Hb in patients with CSF.
\end{abstract}

Key words: coronary slow flow, platelet distribution width.

\section{Introduction}

The phenomenon of coronary slow flow (CSF) is an angiographic clinical entity characterised by delayed distal vessel opacification in the absence of significant epicardial coronary stenosis [1]. Diagnostic criteria for CSF include the absence of coronary atherosclerosis. What is important here is the atherosclerosis plaque, which visually forms significant or non-significant stenosis. Clinical data indicates that CSF is present in approximately $1-7 \%$ of coronary angiography screenings [2], and although not a particularly rare condition, the underlying pathophysiological mechanisms of CSF are poorly understood. Proposed aetiologies for CSF include small vessel disease, microvascular vasomotor dysfunction, diffuse atherosclerosis, endothelial dysfunction, inflammation, increased platelet aggregation, and factors related to anatomic variation [1, 3-6]. Recently-published data associates CSF with poor prognostic outcomes, including cardiac dysfunction, acute coronary syndrome, fatal arrhythmias, and sudden cardiac death [5-8]. Platelet distribution width (PDW) is a direct measure of the variation in platelet size and a marker of platelet activation [9]. $A$ recent study demonstrated a significant association between coronary artery disease (CAD) and PDW. Interestingly, PDW has also been linked to saphenous vein graft patency among patients who underwent coronary artery bypass [10]. Haemoglobin $(\mathrm{Hb})$ is a key determinant of blood viscosity [11]. Red cell distribution width

\section{Corresponding author:}

Ferhat Ozyurtlu MD, Special Grand Medical Hospital, 45030 Manisa, Turkey, phone: +90 530543 61 06, e-mail: fozyurtlu@yahoo.com Received: 31.12.2013, accepted: 13.02.2014. 
(RDW) is a direct measure of the variation in erythrocyte size that is easily measured as a component of routine blood counts [12]. The RDW is a well recognised indicator of chronic inflammation and oxidative stress, and elevated RDW is strongly associated with poor clinical outcomes among patients with CAD $[13,14]$. Neutrophil/ lymphocyte ratio (NLR), derived from the white blood cell count (WBC), is a common prognostic indicator in cardiovascular disease $[15,16]$.

\section{Aim}

The aim of the present study is to evaluate the relationships between CSF, PDW, and other haematological parameters in an effort to identify useful clinical indicators in patients undergoing coronary angiography.

\section{Material and methods}

\section{Patient selection}

A retrospective evaluation of consecutive patients undergoing coronary angiography was conducted. All patients enrolled in the study underwent coronary angiography as a result of chest pain and objective signs of ischaemia during treadmill exercises or myocardial SPECT testing. Routine laboratory and clinical parameters (e.g. diabetes mellitus (DM), hypertension ( $\mathrm{HT}$ ), hypercholesterolemia, tobacco use, family history of cardiovascular disease) were obtained from the patient medical records. Study exclusion criteria included coronary ectasia, mild-severe valve disease, heart failure, anaemia, renal failure, inflammatory diseases, malignancy, peripheral and cerebral arterial disease, thyroid gland dysfunctions (hypo-hyperthyroidism), and LV (left ventricle).

\section{Echocardiography}

All patients underwent transthoracic echocardiography performed using a system $\mathrm{V}$ (Vingmed, GE) device and a $2.5 \mathrm{MHz}$ phased-array transducer. Recordings were made with the patients in the left lateral decubitus position. LV ejection fraction was measured using the modified Simpson's rule according to the most recent guidelines.

\section{Coronary angiography}

Coronary angiography was performed using a GE imaging system (General Electric Advantx LC +, Milwkuee, Wisconsin, US). The standard selective coronary angiography procedure in our clinic includes at least four views of the left coronary system and two views of the right coronary artery using the Judkins technique and 6-French right and left heart catheters and no nitroglycerin. lohexol (GE Healthcare Omnipaque $350 \mathrm{ml}$, Ireland) was used as the contrast agent for all patients and control subjects. Coronary flow rates were measured using the Myocardial
Infarction (TIMI) frame count (TFC) method, with cineangiography at 30 frames per second. Coronary angiograms included at least four images of the left cardiac system and two images of the right cardiac system. These are in the form of cranial and caudal angulations of the left and right oblique images. Images with the best resolution were used for TFC evaluations of the left coronary artery system, and TFC evaluations of the right coronary artery were performed with the left anterior image with cranial angulation. In the cases, invasive haemodynamic evaluations were performed during the coronary angiography. Therefore, haemodynamic changes resulting from catheterisation and vasovagal syndrome could be evaluated with pressure trace; data indicating slow flow as a result of catheterisation and vasovagal syndrome were excluded. Coronary angiograms were assessed independently for objective quantification of coronary flow by two invasive cardiologists blinded to the clinical findings.

\section{Laboratory parameters}

Prior to coronary angiography, eight-hour postprandial venous blood was collected from all patients for routine laboratory testing. Haematological measurements were made using a Mindray Haematology Analyser (BC6800 , China) and were evaluated for complete blood count (CBC). LDL cholesterol analysis was performed using an Olympus AU 2700 Plus Chemistry-ImmunoAnalyser (USA).

\section{Definitions}

Stable angina was defined as discomfort in the chest, jaw, shoulder, back, or arms, typically elicited by exertion or emotional stress, and relieved by rest or nitroglycerin. In accordance with World Health Organization criteria, anaemia was defined as a baseline haemoglobin concentration < $13 \mathrm{mg} / \mathrm{dl}$ in men or $<12 \mathrm{mg} / \mathrm{dl}$ in women. Glomerular filtration rate (GFR) was estimated using the simplified Modification of Diet in Renal Disease Equation [17]. Renal insufficiency was defined as GFR $<60 \mathrm{ml} / \mathrm{min} / 1.732 \mathrm{~m}^{2}$. The TFC was derived according to the methods of Gibson et al. [18]. The first frame was defined as the frame in which radiocontrast agent was first visualised in the ostial region of the coronary artery. The last frame was defined as the frame in which the radiocontrast agent reaches the distal index point of the relevant coronary artery. The $L A D$ is longer than all other coronary arteries; therefore, the corrected TFC (cTFC) is the LAD frame number divided by 1.7. SCF was defined as CTFC greater than two standard deviations from the normal range (40.8 frames for LAD, 29.8 frames for LCX, and 27.3 frames for RCA), while normal coronary was defined as CTFC within two standard deviations of the normal range reported for a particular vessel. All study parameters were reviewed and approved by the Local Ethics Committee. 


\section{Statistical analysis}

The statistical package for social sciences (SPSS, version 15) was used for all data analysis. Continuous data are expressed as mean \pm standard deviation (SD), and categorical data are reported as percentages. The Student's $t$-test was used to compare continuous parametric variables. The $\chi^{2}$ test was used to compare distributions of categorical variables. Cut-off values of PDW and $\mathrm{Hb}$ concentration for the prediction of SCF and their respective sensitivity and specificity values were estimated using receiving operating characteristic (ROC) curve analysis. The threshold of statistical significance was established at $p<0.05$.

\section{Results}

The study population consisted of 6280 consecutive patients undergoing coronary angiography. Out of the total population, 136 patients with SCF were included in the study group. The control group consisted of 152 age-matched subjects with normal coronary angiograms selected consecutively during the same study period as the study group. The same exclusion criteria were applied to the study and control groups. The distribution of cardiovascular risk factors, demographic characteristics, and laboratory parameters in the two groups are shown in Table I. The mean age of the CSF group was $53 \pm 9$ years with a male gender dominance of $61.03 \%$. Among known CAD risk factors, diabetes mellitus and smoking history were more prevalent in the CSF group than in the control group ( $29 \%$ vs. $17 \%, p=0.019$ and $58 \%$ vs. $46 \%$, $p=0.028$, respectively, Table I). The PDW (16.6 $\pm 0.7 \%$ vs. $16.4 \pm 0.6 \%, p=0.002)$, NLR (3.1 \pm 3.4 vs. $2.4 \pm 1.1$, $p=0.027)$, haemoglobin $(14.1 \pm 1.3 \mathrm{~g} / \mathrm{dl}$ vs. $14.7 \pm 1.1 \mathrm{~g} / \mathrm{dl}$, $p<0.001)$, and RDW ( $13.6 \pm 0.7 \%$ vs. $14.1 \pm 2.8 \%, p=0.026)$ were significantly increased in the CSF group relative to the control group (Table II). A PDW of 16.15 predicted CSF with a sensitivity of $83 \%$ and a specificity of $40 \%$ (ROC AUC: $0.618,95 \% \mathrm{Cl}: 0.554-0.683, p=0.001$ ) (Figure $1 \mathrm{~A}$ )
Table I. Distribution of baseline characteristic of all patients

\begin{tabular}{lccc} 
Variables & $\begin{array}{c}\text { Normal flow } \\
(n=152)\end{array}$ & $\begin{array}{c}\text { Slow flow } \\
(n=136)\end{array}$ & $\begin{array}{c}\text { Value } \\
\text { of } \boldsymbol{p}\end{array}$ \\
\hline Age [years] & $52 \pm 10$ & $53 \pm 9$ & 0.379 \\
\hline Male gender, $n(\%)$ & $71(47)$ & $83(61)$ & 0.015 \\
\hline Family history, $n(\%)$ & $34(22)$ & $33(24)$ & 0.704 \\
\hline Smoking, $n(\%)$ & $46(30)$ & $58(43)$ & 0.028 \\
\hline Diabetes mellitus, $n(\%)$ & $17(11)$ & $29(21)$ & 0.019 \\
\hline Hyperlipidaemia, $n(\%)$ & $51(34)$ & $62(46)$ & 0.037 \\
\hline Hypertension, $n(\%)$ & $58(38)$ & $43(32)$ & 0.246 \\
\hline LDL-c $[\mathrm{mg} / \mathrm{dl}]$ & $116 \pm 25$ & $112 \pm 33$ & 0.476 \\
\hline SBP $[\mathrm{mm}$ Hg] & $124 \pm 17$ & $121 \pm 16$ & 0.008 \\
\hline DBP $[\mathrm{mm}$ Hg] & $76 \pm 11$ & $75 \pm 9$ & 0.17 \\
\hline Heart rate $[\mathrm{bpm}]$ & $77 \pm 12$ & $74 \pm 13$ & 0.046 \\
\hline TIMI frame count & $23 \pm 2.8$ & $33 \pm 6.4$ & $<0.001$ \\
\hline LAD cTFC & $26.8 \pm 4.3$ & $38.1 \pm 13.9$ & $<0.001$ \\
\hline LCX TFC & $20.9 \pm 3.7$ & $30.2 \pm 9.5$ & $<0.001$ \\
\hline RCA TFC & $20.7 \pm 3.6$ & $33.3 \pm 11.5$ & $<0.001$
\end{tabular}

Values are mean (SD), LDL-C-low density lipoprotein cholesterol, SBP - systolic blood pressure, DBP - diastolic blood pressure, $L A D$ - left anterior descending coronary artery, $L C X$ - left circumflex coronary artery, $R C A$ - right coronary artery, TFC - TIMI frame count, CTFC - corrected TIMI frame count

Table II. Distribution of the haematological parameters of all cases

\begin{tabular}{lccc} 
Variables & $\begin{array}{c}\text { Normal flow } \\
(\boldsymbol{n}=152)\end{array}$ & $\begin{array}{c}\text { Slow flow } \\
(\boldsymbol{n}=136)\end{array}$ & $\begin{array}{c}\text { Value } \\
\text { of } \boldsymbol{p}\end{array}$ \\
\hline Haemoglobin $[\mathrm{g} / \mathrm{dl}]$ & $14.1 \pm 1.3$ & $14.7 \pm 1.1$ & $<0.001$ \\
\hline WBC & $7.6 \pm 1.7$ & $7.9 \pm 2.4$ & 0.289 \\
\hline MCV & $87.2 \pm 7.6$ & $88.3 \pm 5.6$ & 0.164 \\
\hline Platelet $\left[\times 1000 / \mathrm{mm}^{3}\right]$ & $244 \pm 46$ & $244 \pm 66$ & 0.989 \\
\hline RDW $[\%]$ & $13.6 \pm 0.7$ & $14.1 \pm 2.8$ & 0.026 \\
\hline MPV [fl] & $8.5 \pm 1.1$ & $8.7 \pm 1.1$ & 0.079 \\
\hline PDW [\%] & $16.4 \pm 0.6$ & $16.6 \pm 0.7$ & 0.002 \\
\hline NLR & $2.4 \pm 1.1$ & $3.1 \pm 3.4$ & 0.027
\end{tabular}

WBC - white blood cell count, MCV - mean corpuscular volume, RDW - red blood cell distribution width, MPV - mean platelet volume, PDW - platelet distribution width, NLR - neutrophil/lymphocyte ratio
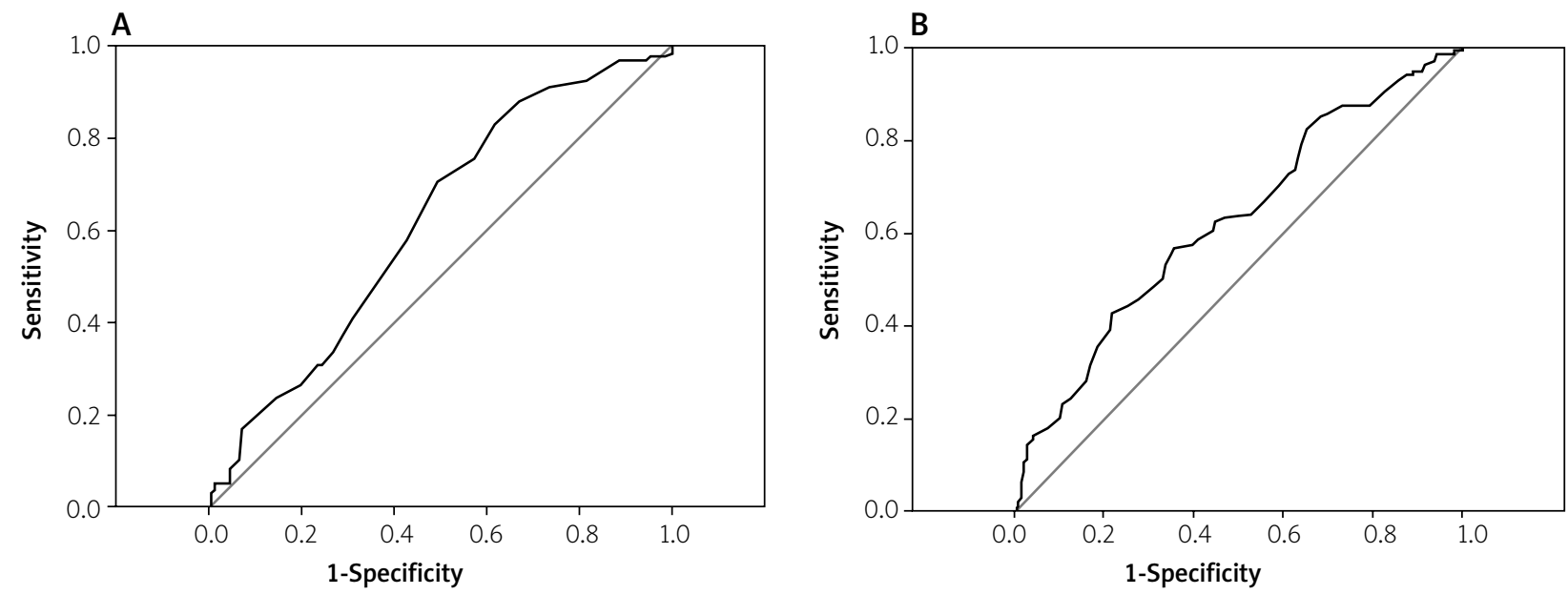

Figure 1. Receiver operating characteristics (ROC) analysis of PDW (A) and $\mathrm{Hb}(\mathbf{B})$ concentration 
and an $\mathrm{Hb}$ concentration of $13.75 \mathrm{~g} / \mathrm{dl}$ predicted CSF with a sensitivity of $73 \%$ and a specificity of $42 \%$ (ROC AUC: $0.625,95 \% \mathrm{Cl}: 0.561-0.690, p<0.001)$ (Figure $1 \mathrm{~B}$ ).

\section{Discussion}

In this study we examined the relationship between CSF, PDW, and other haematological parameters. The CSF was independently associated with PDW, Hb, NLR, and RDW. Moreover, our study data demonstrate that PDW $>16.15 \%$ and $\mathrm{Hb}>13.75 \mathrm{~g} / \mathrm{dl}$ predict the presence of CSF with sensitivities and specificities of $83 \%$ and $73 \%$, and $40 \%$ and $42 \%$, respectively.

A limited number of studies have been performed examining the pathophysiology of CSF since the first description of the disease by Tambe et al. in 1972. Several competing hypotheses explaining the aetiology of CSF have emerged. These include atherosclerosis and increased thrombogenic activity. Inflammation plays an important role in the onset, development, and progression of atherosclerosis. Atherosclerosis is itself considered an inflammatory disease. Microvascular atherosclerosis may contribute to the development of CSF. Recent studies have demonstrated the relationship between CSF and inflammation.

Vagdatli et al. [19] have proposed that PDW is a more specific indicator of platelet activation than MPV in the absence of platelet swelling. Elevated PDW directly measures the variability in platelet size during platelet distension and serves as a marker of platelet activation [20]. Increased platelet number, size, and the presence of pseudopodia may influence PDW. Khandekar et al. reported a significant elevation of PDW among patients with acute myocardial infarction and unstable angina pectoris [21]. Our study data demonstrate a significant association between PDW and CSF. A study conducted by Jindal et al. identified a significant association between PDW and microvascular dysfunction among diabetic patients [22]. The CSF may be a symptom of microvascular and circulatory dysfunction. Numerous factors contribute to microvascular and circulatory dysfunction, including coronary microvascular imbalance and increased tonus, endothelial thickening of small vessels and endothelial nitric oxide imbalance, and blood viscosity. $\mathrm{Hb}$ concentration is a determinant of blood viscosity, and a significant relationship between CSF and $\mathrm{Hb}$ concentration has been demonstrated previously [11]. The SCF patients frequently present with elevated blood viscosity [23]. Similar to atherosclerosis, blood viscosity is elevated in SCF patients as a result of increased platelet adhesion to the subendothelium, elevated protein infiltration into the arterial wall, and alterations to local shear forces [24]. Our data supports a significant relationship between CSF and haemoglobin concentration, a component of blood viscosity.

The NLR is associated with the onset and progression of atherosclerosis in the coronary arteries [25], and re- cent studies suggest that NLR is an excellent indicator of cardiovascular disease $[15,16,26]$. As a result, NLR has emerged as a new prognostic indicator [16, 27]. Among patients with acute coronary syndrome, neutrophils are functionally activated, and the presence of localised neutrophil infiltration in atherosclerotic lesions has been documented, suggesting that neutrophils play a role in the mediation and destabilisation of atherosclerotic plaques [28]. The present study demonstrates a significant correlation between the presence of CSF and NLR, an inflammatory marker linked to CAD and atherosclerosis.

Chronic inflammation and neuro-humoral activation can act synergistically to elevate RDW, enhancing the atherosclerotic process [29]. The RDW is an independent predictor of mortality and coronary morbidity among patients with myocardial infarction $[13,14]$. Similar to the report by Kalay et al. [25], the present study confirms a significant association between CSF and RDW. A recent study by Akpinar et al. involving a comparable number of patients reported elevated PDW, RDW, and NLR similar to our CSF study group [30].

The relationship between cardiovascular disease and increased platelet activity is well known. In this study, we found a significant relationship between CSF and PDW, an established indicator of platelet activity. In addition, we detected a significant relationship between CSF and $\mathrm{NLR}$, an indicator of systemic inflammation, and $\mathrm{Hb}$ concentration, a component of blood viscosity. These predictive parameters are easily measured and are inexpensive in routine clinical practice. In case, it is supported in other studies; in patient with chest pain care the high value of PDW can be ranked in CSF diagnosis algorithm.

In this study we evaluated the coronary arteries using coronary angiography. Although it is well known that IVUS provides a more precise evaluation of coronary atherosclerosis, we were unable to perform intravascular ultrasound (IVUS) assessment. In addition, the study data is reflective of the cross-sectional design and may not reflect the long-term clinical status of the patients.

\section{Conclusions}

Our study data support a statistically significant association between PDW and CSF. Further studies of CSF will continue to advance our understanding of its etiopathology.

\section{References}

1. Wang X, Nie SP. The coronary slow flow phenomenon: characteristics, mechanisms and implications. Cardiovasc Diagn Ther 2011; 1: 37-43.

2. Mangieri E, Macchiarelli G, Ciavolella M, et al. Slow coronary flow: clinical and histopathological features in patients with otherwise normal epicardial coronary arteries. Cathet Cardiovasc Diagn 1996; 37: 375-81. 
3. Sezgin AT, Sigirci A, Barutcu I, et al. Vascular endothelial function in patients with slow coronary flow. Coron Artery Dis 2003; 14: 155-61.

4. Gokce M, Kaplan S, Tekelioglu Y, et al. Platelet function disorder in patients with coronary slow flow. Clin Cardiol 2005; 28: 145-8.

5. Pekdemir H, Cin VG, Cicek D, et al. Slow coronary flow may be a sign of diffuse atherosclerosis. Contribution of FFR and IVUS. Acta Cardiol 2004; 59: 127-33.

6. Beltrame JF, Limaye SB, Horowitz JD. The coronary slow flow phenomenon - a new coronary microvascular disorder. Cardiology 2002; 97: 197-202.

7. Nurkalem Z, Gorgulu S, Uslu N, et al. Longitudinal left ventricular systolic function is impaired in patients with coronary slow flow. Int I Cardiovasc Imaging 2009; 25: 25-32.

8. Saya S, Hennebry TA, Lozano P, et al. Coronary slow flow phenomenon and risk for sudden cardiac death due to ventricular arrhythmias: a case report and review of literature. Clin Cardiol 2008; 31: 352-5.

9. Herve P, Humbert M, Sitbon O, et al. Pathobiology of pulmonary hypertension: the role of platelets and thrombosis. Clin Chest Med 2001; 22: 451-8.

10. Ege MR, Guray U, Guray Y, et al. Platelet distribution width and saphenous vein disease in patients after CABG: association with graft occlusion. Herz 2013; 38: 197-201.

11. Wang S, Zhang Y, Cheng Y, et al. Correlation between the hematocryte and slow coronary flow. Clin Hemorheol Microcirc 2013 Jan 2.

12. Tonelli M, Sacks F, Arnold M, et al. Relation between red blood cell distribution width and cardiovascular event rate in people with coronary disease. Circulation 2008; 117: 163-8.

13. Dabbah S, Hammerman H, Markiewicz W, et al. Relation between red cell distribution width and clinical outcomes after acute myocardial infarction. Am J Cardiol 2010; 105: 312-7.

14. Polat N, Yildiz A, Oylumlu M, et al. Relationship between red cell distribution width and the GRACE Risk score with in-hospital D eath in patients with acute coronary syndrome. Clin Appl Thromb Hemost 2014; 20: 577-82.

15. Kaya H, Ertas F, Islamoglu Y, et al. Association between neutrophil to lymphocyte ratio and severity of coronary artery disease. Clin Appl Thromb Hemost 2014; 20: 50-4.

16. Acet $\mathrm{H}$, Ertaş F, Akıl MA, et al. New inflammatory predictors for non-valvular atrial fibrillation: echocardiographic epicardial fat thickness and neutrophil to lymphocyte ratio. Int I Cardiovasc Imaging 2014; 30: 81-9.

17. Stevens LA, Coresh J, Greene T, et al. Assessing kidney function measured and estimated glomerular filtration rate. N Engl I Med 2006; 354: 2473-83.

18. Gibson CM, Cannon CP, Daley WL, et al. TIMI frame count: a quantitative method of assessing coronary artery flow. Circulation 1996; 93: 879-88.

19. Vagdatli E, Gounari E, Lazaridou E, et al. Platelet distribution width: a simple, practical and specific marker of activation of coagulation. Hippokratia 2010; 14: 28-32.

20. Herve P, Humbert M, Sitbon O, et al. Pathobiology of pulmonary hypertension: the role of platelets and thrombosis. Clin Chest Med 2001; 22: 451-8.

21. Khandekar MM, Khurana AS, Deshmukh SD, et al. Platelet volume indices in patients with coronary artery disease and acute myocardial infarction: an Indian scenario. J Clin Pathol 2006; 59: 146-9.
22. Jindal S, Gupta S, Gupta R, et al. Platelet indices in diabetes mellitus: indicators of diabetic microvascular complications. Hematology 2011; 16: 86-9.

23. Ergun-Cagli K, lleri-Gurel E, Ozeke O, et al. Blood viscosity changes in slow coronary flow patients. Clin Hemorheol Microcirc 2011; 47: 27-35.

24. Lowe GD. Blood rheology in arterial disease. Clin Sci (Lond) 1986; 71: 137-46.

25. Kalay N, Dogdu O, Koc F, et al. Hematologic parameters and angiographic progression of coronary atherosclerosis. Angiology 2012; 63: 213-7.

26. Hansson GK. Inflammation, atherosclerosis, and coronary artery disease. N Engl J Med 2005; 352: 1685-95.

27. Gibson PH, Cuthbertson BH, Croal BL, et al. Usefulness of neutrophil/lymphocyte ratio as predictor of new-onset atrial fibrillation after coronary artery bypass grafting. Am J Cardiol 2010; 105: 186-91.

28. Naruko T, Ueda M, Haze K, et al. Neutrophil infiltration of culprit lesions in acute coronary syndromes. Circulation 2002; 106: 2894-900.

29. Packard RR, Libby P. Inflammation in atherosclerosis: from vascular biology to biomarker discovery and risk prediction. Clin Chem 2008; 54: 24-38.

30. Akpinar I, Sayin MR, Gursoy YC, et al. Plateletcrit and red cell distribution width are independent predictors of the slow coronary flow phenomenon. J Cardiol 2014; 63: 112-8. 